\title{
On the Technical Composition and Development Trend of Fieldbus
}

\author{
Shi Honghao
}

China Shipping Petrochemical Co., Ltd. Dongfang City, Hainan Province 572600

\begin{abstract}
Fieldbus technology is the forefront technology of digital communication in contemporary industry. It is the integration of computer technology, communication technology and automation control technology. It is also the embodiment of information technology and measurement technology in the information age. Fieldbus technology is a pioneer in the era of industrial digital communications, and its emergence is causing an unprecedented technological revolution in the field of industrial control. In the future development of fieldbus technology, China should combine the actual situation of social development, establish a long-term development goals, and humbly learn from the advanced countries of the world's advanced experience, access to foreign countries advanced technology and information to master the maturity of foreign development technology. Based on this paper, the technical structure and development trend of fieldbus are analyzed.
\end{abstract}

Key words: Fieldbus technology; composition; development trend

\section{The Basic Concept and Features of Field Bus}

\subsection{The Basic Concept of Field Bus}

Fieldbus is a kind of new digital communication technology which originated from the 1980s. It can be applied to the corresponding production and construction site, the electric power dispatching work and the multi-node digital communication work between the microcomputer equipment, because of its unique application scope and use, in the specific use of the process it is also known as a full range of digital control system of the underlying network center. Fieldbus can effectively handle field devices, such as the implementation of controllers, institutions, intelligent instruments and other digital communications between, and in the field control equipment and advanced control systems to achieve better information between the transfers. Fieldbus due to good reliability, economical and practical and easy to deconstruct the advantages of making it in many areas can be widely used.

\subsection{The Characteristics of Field Bus Technology}

(1) Operability and mutual help

In the past, China's power science and technology has not yet fully developed the period of power system is a self-enclosed ring system, which can only unilaterally pass the corresponding communication information, and cannot achieve the corresponding mutual aid and mutual function. In the field bus technology under the guidance of China's power system can gradually break through the ring structure, and gradually towards the double-sided recycling structure of the style of development, so that you can achieve a certain operation, better achieve mutual aid and mutual goals.

\section{(2) Intelligent}

In the past, the DCS must be used in the following three basic media, namely: the operation center, the control station and the scene of the equipment connection can be run and get used, while the DCS operation mode is more common single-phase transmission form. In the process of digital signal transmission will inevitably be due to external factors interfere with the corresponding error and the intelligence of fieldbus technology can make up for this shortcoming, which is usually taken in the FCS is a two-way digital signal transmission form, which can be applied to the measurement and compensation calculation applied to the scene of the equipment operation for being better to achieve automatic control and control functions.

\section{Adaptability}

According to the basic concept of field bus, we can see that it is a kind of ground control network, mainly for the field environment control. So it also has a strong adaptability in the course of its use, and it also supports the corresponding twisted pair, optical cable, infrared and power lines, and in the use of the process to resist some of the necessary external interference.

\section{The Main Advantages of Field Bus Technology}

\subsection{Reduce Maintenance Costs}

In the field bus applications, you can install, maintain, wiring power system in the process, save a lot of cost. In the power transmission which can track the signal 
in real time, compared to the traditional control system, effectively reducing the cost. In the field bus technology, usually use a unified power equipment, can save a lot of power system manpower, material and financial resources, so that the power system installation project complexity issues are resolved. Moreover, by using the fieldbus technology in the automatic fault diagnosis and troubleshooting functions, which can reduce the workload of maintenance personnel, thereby reducing maintenance costs.

\subsection{To Improve Maintainability}

The application of fieldbus technology is very important. Between the power transmissions equipment, can effectively reduce the number of connections and the number of connections, so as to better ensure the stability of the power system. If a point in the system has failed, fieldbus technology can provide greater convenience during the inspection and analysis of the cause of the failure. Moreover, after the application of field bus technology, it can have online fault diagnosis, online troubleshooting and other functions, making the system maintainability has been greatly improved.

\subsection{To Promote Digital Communications Automation}

If the signal is to be propagated, it can be converted by fieldbus technology to form the corresponding digital signal and then use the digital communication function to transmit these signals. Through this automated digital communication, it is possible to discover the faults that are difficult to be found in the past information transmission, so as to solve and deal with the failure in time to avoid the unsafe operation of the system in the fault condition, strengthen the communication and contact between the customers. Multi-site to achieve a good twoway communication between, so as to better ensure the quality of communication and communication speed.

\section{The Field Bus Classification}

\subsection{Foundation Fieldbus}

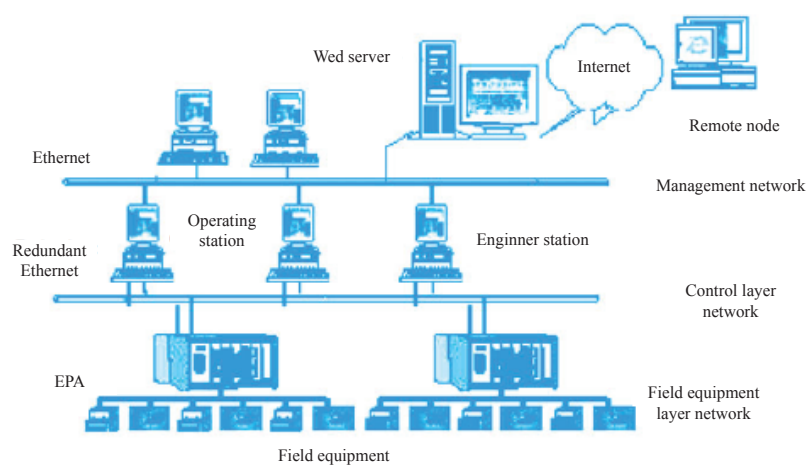

Foundation Field bus, referred to as FF, which is in the field of process automation has been widely supported and has good prospects for the development of technology. The predecessor of the United States Fisher-Rousemount company headed by the United States Foxboro, Yokogawa, ABB, Siemens and other 80 companies to develop ISP agreement and led by Honeywell, the joint
European companies such as 150 companies developed WordFIP agreement. The pressure on the user, the two groups merged in September 1994, set up a field bus foundation, committed to the development of a unified international fieldbus agreement. It is based on the ISO / OSI open system interconnection model, taking its physical layer, data link layer, application layer as the corresponding level of the FF communication model, and adding the user layer at the application layer.

The Foundation fieldbus is divided into two low-speed $\mathrm{H} 1$ and high-speed $\mathrm{H} 2$ communication rates. H1 transmission rate of $3125 \mathrm{Kbps}$, the communication distance of up to $1900 \mathrm{~m}$ (plus repeater extension), can support the bus power supply, support intrinsically safe explosion-proof environment. $\mathrm{H} 2$ transmission rate of $1 \mathrm{Mbps}$ and $25 \mathrm{Mbps}$ two, the communication distance of $750 \mathrm{~m}$ and $500 \mathrm{~m}$. Physical transmission media can support than the twisted pair, fiber optic cable and wireless transmission, the agreement in line with IEC1158-2 standard. The physical medium of the transmission signal using Manchester coding, each sent the data center or positive jump, or negative jump. A positive transition represents 0 , and a negative transition represents 1 , so that the serial data bitstream has sufficient positioning information to keep both timestamps sent. The receiver can judge the " 1 ", " 0 " state of the data according to the polarity of the jump, or locate the data according to the center of the data.

\subsection{CAN Control Network Bus}

CAN is the control network Control Area Network referred to, the first by the German company BOSCH introduced for automotive internal measurement and implementation of the data between the components of communication. Its bus specification is now ISO international standards organizations to develop international standards, has been Motorola, Intel, Philips, Siemens, NEC and other companies support, has been widely used in the field of discrete control.

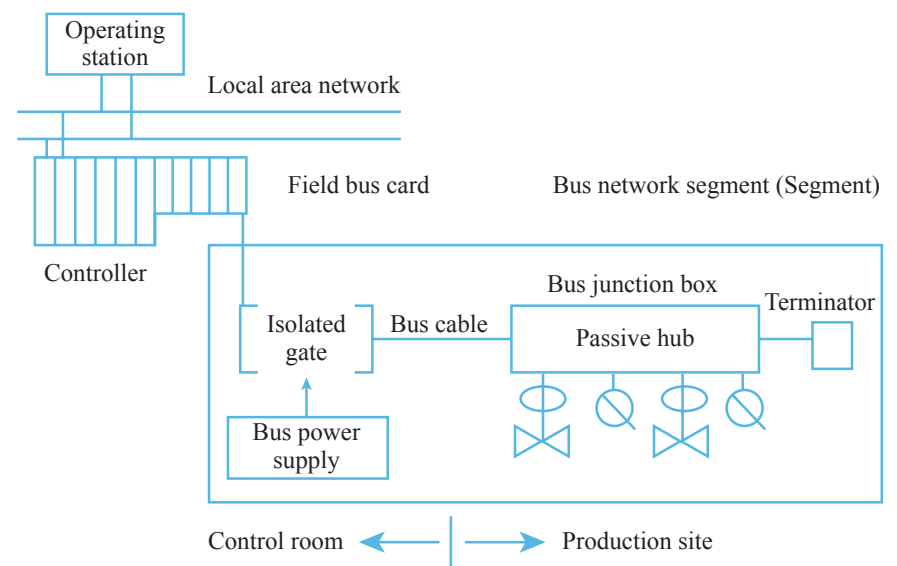

The CAN protocol is also based on the open system interconnection model of the International Standards Organization. However, the model structure is only 3 layers, only the physical layer, the data link layer and the top layer application layer of the OSI. The signal transmission medium for the twisted pair, the communication rate 
of up to $1 \mathrm{Mbps} / 40 \mathrm{~m}$, direct transmission distance up to $10 \mathrm{~km} / \mathrm{kbps}$, which can be mounted up to 110 devices.

CAN supports multi-master mode, any node on the network at any time to actively send information to other nodes, support point to point, point to multipoint and global broadcast mode to receive/send data. It uses bus arbitration technology. When several nodes simultaneously transmit information on the network, nodes with high priority can continue to transmit data, while nodes with low priority take the initiative to stop sending, thus avoiding the bus collision.

\subsection{HART Bus}

HART is an abbreviation for Highway Addressable Remote Transducer. Was first developed by Rosemount and in 1993 established the HART Communications Foundation. This is called an addressable remote sensing highspeed channel open communication protocol, which is characterized by the existing analog signal transmission line to achieve digital communications, analog systems to the digital system transition process of industrial process control transition products. The current transition period has a strong market competitiveness and has been a better development.

The HART communication model consists of three layers: the physical layer, the data link layer, and the application layer. The physical layer uses FSK (Frequency Shift Keying) technology in the $4 \sim 20 \mathrm{~mA}$ analog signal superimposed on a frequency signal, the frequency signal using Bel1202 international standard. Data transmission rate of $1200 \mathrm{bps}$, logic " 0 " signal frequency of $2200 \mathrm{~Hz}$, logic " 1 " signal transmission frequency of $1200 \mathrm{~Hz}$. At present it has a certain market share.

\section{The Impact and Application of Field Bus on the Au- tomation}

\subsection{To Promote Industrial Control from the Control Room} to the Scene

Field bus for automation instruments and systems of the most direct and greatest impact is to achieve a variety of control functions from the control room to the scene changes, fundamentally changed the factory control system structure. Digital instrumentation will be replaced by analog meters, the current DCS control function will be replaced by the FCS system function. Fieldbus involved in a variety of new concepts, which will continue to promote the field instrument detection and control functions of the update. To this end, the government and the decision-making agencies should actively encourage the instrument manufacturers and the majority of users to analyze research and development applications on-site bus for all sectors of our national economy to provide new automation equipment, the revitalization of China's instrument industry. Especially in the face of the current foreign instrumentation company products into the market competition in China's hot situation, China's instrument companies are facing difficulties, to promote the development of fieldbus instruments and systems, will promote China's instrument industry and product structure adjustment, so that our country's instrument industry in the technology and market driven by a competitive advantage.

\subsection{To Achieve the Transmission Signal Digitization}

First, the fieldbus will completely replace the $4 \sim 20 \mathrm{~mA}$ DC contact signal, to achieve digital transmission signal, more convenient for field wiring, reducing the cost of nearly $70 \%$ of the field wiring. Second, the field bus can control, alarm, trend analysis and other advanced features to the field instrument, reducing the $\mathrm{D} / \mathrm{A}$ and $\mathrm{A} / \mathrm{D}$ conversion, the upper system is simplified. Finally, the fieldbus to lay the foundation for the opening of the system to achieve the automation of the instrument from the closed system to the open system changes from the analog-digital hybrid technology to all digital technology changes, the manufacturers under the same standard equipment through interconnection Interoperability and interchangeable use, which greatly facilitates the exchange of information between users. In addition, the use of fieldbus to complete the underlying communications, system redundancy and fault diagnosis and fault tolerance enhancements, greatly improving the system reliability, security, for its wider areas of application to create the conditions. To this end, this paper stated that the Government should actively organize the establishment of "China Fieldbus Association" to form a business, large groups, research institutes and universities and other forces as one of the cohesion, work together on the field bus technology and product development and application to achieve with the international community will always organize the convergence and interaction, pay close attention to the development of international standards for fieldbus to better guide our fieldbus technology development and improvement. Such as the national key arrangements for several DCS application skilled and strong economic strength of the chemical industry and instrumentation research, production sector group, into the International Fieldbus Foundation, and strive to become its test base, to obtain the actual test of the initial data for the development of China A new generation of instruments to lay the foundation.

\section{The Development Trend of Field Bus Technology}

\subsection{The Development Trend of Field Bus}

In recent years, industrial Ethernet technology has been rapid development, this standard was originally used in the field of IT, is also in the information network expansion to achieve a wide range of development. Compared to other industrial automation technologies, industrial Ethernet technology with its increasingly perfect performance and rapid reduction in the cost of absolute advantage, which makes a lot of industrial automation companies will focus more on the use of Ethernet resources, so the new generation of industrial automation network is bound to be based on the establishment of Ethernet, Ethernet technology has become a hotspot to support industrial development. Analysis of the direction of the de- 
velopment of bus technology, all the current field bus will achieve the transition to Ethernet, many fieldbus-based control system will eventually establish a connection with Ethernet, to meet certain conditions, but also to achieve the connection with the Internet. For industrial control systems, industrial Ethernet is a major development direction, the current process monitoring layer of industrial Ethernet for a large number of applications, its application in the field layer is mainly on the use of fieldbus technology to reflect.

\subsection{The Development of Field Bus Path}

First, the government departments and the decision-making agencies should give high priority to the development of fieldbus technology, as soon as possible to respond, to promote the development of technology to the direction of the country will rise. Second, the relevant departments in the whole society to carry out industrial propaganda, so that the instrument manufacturers and the majority of users on the field bus technology has a full understanding and understanding, lay a solid foundation for the technology of science and technology research, research and development and application. Third, the establishment of a number of field bus association, to the enterprise as the main body, dependent on the support of large enterprise groups, allowing users to participate in a wide range, accelerate the development of technology, expand product development and application scale, open pattern, in line with the international related work to carry out the guid- ance. Fourth, the absorption of human, material and financial resources and other resources to field bus-based intelligent network automation instrument to develop, from scientific and technological research product development, technological transformation and industry work to promote the relevant units to provide key support, the establishment of open automation system production base, the formation of appropriate economies of scale.

In short, nowadays, the field bus technology has been widely concerned, it is a hot research topic, and to further strengthen its research is of great significance which needs our attention.

\section{References}

[1] Chen Xiaoqin. LLC Resonant Converter Digital Parallel Technology Research [D]. Guangdong University of Technology, 2015.

[2] Shao Qing. Study and Implementation of Foundation Field Bus Data Communication Technology [D]. Daqing Petroleum Institute, 2005.

[3] Zhang Shanshan. Research and Implementation of Configuration Technology Based On Field Bus [D]. Daqing Petroleum Institute, 2005.

[4] Wang Zheng. Fieldbus Communication Technology Research and Implementation [D]. Daqing Petroleum Institute, 2004. 